\title{
PROOF OF A FORMULA OF LIOUVILLE
}

WALTER H. GAGE

1. Introduction. In his summary of the formulas stated without proof in Liouville's famous series of articles on the theory of numbers, Dickson [1] ${ }^{1}$ remarked that no proof had been published for (Q) of the Sixth Article [2]. This formula, however, was later derived by Bell [3], who obtained it by paraphrase, a general method which he applied to various identities between elliptic, abelian, and theta functions to obtain some of Liouville's formulas and others of new kinds.

Bell noted that among all of Liouville's, formula ( $Q$ ) is unique, as "it is the only one which depends immediately upon the addition theorems for the elliptic functions." For this reason, together with the fact that it is the only elementary proof so far published, the derivation of formula (Q) presented below may be of interest.

2. Notation. Throughout this paper $\left(d_{1}, \delta_{1}, d_{2}, \delta_{2}, d_{3}, \delta_{3}\right)$ and $\left(d_{1}^{\prime}, \delta_{1}^{\prime}, d_{2}^{\prime}, \delta_{2}^{\prime}, d_{3}^{\prime}, \delta_{3}^{\prime}\right)$ denote integer solutions for $n$, a fixed positive integer, in the forms

$$
\begin{gathered}
n=d_{1} \delta_{1}+d_{2} \delta_{2}+d_{3} \delta_{3}, \\
n=d_{1}^{\prime} \delta_{1}^{\prime}+d_{2}^{\prime} \delta_{2}^{\prime}+d_{3}^{\prime} \delta_{3}^{\prime}
\end{gathered}
$$

respectively, each of the $d_{i}, \delta_{i}, d_{i}^{\prime}, \delta_{i}^{\prime}(i=1,2,3)$ being positive unless otherwise specified. Further, $F(x, y)$ denotes a function which takes a single definite value for each integral pair $(x, y)$, satisfies the conditions

$$
\begin{aligned}
F(-x, y) & =-F(x, y)=F(x,-y)=F(y, x), \\
F(0, y) & =0=F(x, 0),
\end{aligned}
$$

but is otherwise general in the widest sense.

For the sake of brevity, certain expressions are denoted by single letters with subscripts. These are as follows:

$$
\begin{aligned}
S_{1}= & d_{3}\left\{F\left(d_{1}+d_{2}+d_{3}, d_{1}+d_{2}\right)-F\left(d_{1}-d_{2}-d_{3}, d_{1}-d_{2}\right)\right\} \\
& +\delta_{2}\left\{F\left(\delta_{1}-\delta_{3}, \delta_{1}+\delta_{2}-\delta_{3}\right)-F\left(\delta_{1}+\delta_{3}, \delta_{1}-\delta_{2}+\delta_{3}\right)\right\}, \\
S_{2}= & \left(d_{3}-d_{2}\right)\left\{F\left(d_{1}+d_{3}, d_{1}+d_{2}\right)-F\left(d_{1}-d_{3}, d_{1}-d_{2}\right)\right\} \\
& +\left(\delta_{2}+\delta_{3}\right)\left\{F\left(\delta_{1}-\delta_{3}, \delta_{1}+\delta_{2}\right)-F\left(\delta_{1}+\delta_{3}, \delta_{1}-\delta_{2}\right)\right\},
\end{aligned}
$$

Received by the editors August 4, 1947.

1 Numbers in brackets refer to the references cited at the end of the paper. 


$$
\begin{aligned}
S_{3}= & d_{3}\left\{F\left(d_{1}+d_{2}+d_{3}, d_{1}+d_{2}\right)-F\left(d_{1}+d_{2}, d_{1}+d_{2}-d_{3}\right)\right\} \\
& +\delta_{3}\left\{F\left(\delta_{2}-\delta_{1}, \delta_{2}-\delta_{1}+\delta_{3}\right)-F\left(\delta_{2}-\delta_{1}-\delta_{3}, \delta_{2}-\delta_{1}\right)\right\}, \\
S_{4}= & d_{3}\left\{F\left(d_{1}+d_{3}, d_{1}\right)-F\left(d_{1}, d_{1}-d_{3}\right)\right\} \\
& +\delta_{3}\left\{F\left(\delta_{2}, \delta_{2}+\delta_{3}\right)-F\left(\delta_{2}-\delta_{3}, \delta_{2}\right)\right\} .
\end{aligned}
$$

When each $d_{i}, \delta_{i}$ is replaced by the corresponding $d_{i}^{\prime}, \delta_{i}^{\prime}$ the resulting expressions are denoted by $S_{1}^{\prime}, S_{2}^{\prime}, S_{3}^{\prime}, S_{4}^{\prime}$ respectively, and when $F(x, y)$ is replaced by $\phi(x, y)$, to be defined later, they are likewise denoted by $\bar{S}_{1}, \bar{S}_{2}, \bar{S}_{3}, \bar{S}_{4}$.

The symbol $\sum$ indicates that the expression $S_{i}, S_{i}^{\prime}$ which it precedes is to be summed over integer solutions of $n$ in the form (1), (2) respectively. Any restrictions placed on these solutions are indicated in a square bracket immediately following the summand.

3. Fundamental formulas. It is necessary to derive two fundamental formulas. by

Because of the one-one correspondences between (1) and (2) given

(4) $d_{1}=d_{1}^{\prime}, \delta_{1}=\delta_{1}^{\prime}, d_{2}=d_{2}^{\prime}, \delta_{2}=\delta_{2}^{\prime}, d_{3}=d_{3}^{\prime}, \delta_{3}=\delta_{3}^{\prime}$,

(5) $d_{1}^{\prime}=d_{1}, \delta_{1}^{\prime}=\delta_{1}, d_{2}^{\prime}=d_{2}, \delta_{2}^{\prime}=\delta_{2}+\delta_{3}, d_{3}^{\prime}=d_{3}-d_{2}, \delta_{3}^{\prime}=\delta_{3}$,

it is clear that

$$
\begin{aligned}
\sum S_{1} & {\left[d_{2} \equiv \delta_{3}(\bmod 2), \delta_{2} \equiv d_{3}(\bmod 2)\right] } \\
& =\sum S_{1}^{\prime}\left[d_{2}^{\prime} \equiv \delta_{3}^{\prime}(\bmod 2), \delta_{2}^{\prime} \equiv d_{3}^{\prime}(\bmod 2)\right] \\
& =\sum S_{2}\left[d_{2} \equiv \delta_{3}(\bmod 2), \delta_{2} \equiv d_{3}(\bmod 2),\right. \\
& \left.d_{3}>d_{2}, \delta_{2}+\delta_{3}>0\right],
\end{aligned}
$$

where, in the last sum, $\delta_{2}$ is not restricted to positive values.

As a consequence of (3), $S_{2}$ in the final sum of (6) is invariant for $\delta_{2}>0$, when $d_{2}, \delta_{2}, d_{3}, \delta_{3}$ are replaced by $d_{3}, \delta_{3}, d_{2}, \delta_{2}$ respectively. Hence

$$
\begin{aligned}
\sum & S_{2}\left[d_{2} \equiv \delta_{3}(\bmod 2), \delta_{2} \equiv d_{3}(\bmod 2), d_{3}>d_{2}, \delta_{2}+\delta_{3}>0, \delta_{2}>0\right] \\
= & \sum S_{2}\left[d_{2} \equiv \delta_{3}(\bmod 2), \delta_{2} \equiv d_{3}(\bmod 2), d_{2}>d_{3}, \delta_{2}+\delta_{3}>0, \delta_{2}>0\right] \\
= & 2^{-1} \sum\left\{S_{2}\left[d_{2} \equiv \delta_{3}(\bmod 2), \delta_{2} \equiv d_{3}(\bmod 2)\right]\right. \\
& \left.\quad-S_{2}\left[d_{2} \equiv \delta_{2} \equiv d_{3} \equiv \delta_{3}(\bmod 2), d_{2}=d_{3}\right]\right\} .
\end{aligned}
$$

Similarly, $S_{2}$ in the final sum of (6) for $\delta_{2}<0$ goes into $-S_{2}$, with no change in the restrictions, when $d_{1}, \delta_{1}, d_{2}, \delta_{2}, d_{3}, \delta_{3}$ are replaced by $\delta_{1}, d_{1},-\delta_{2},-d_{2}, \delta_{3}, d_{3}$ respectively. For $\delta_{2}<0$, the final sum in (6) is therefore zero. Hence it follows from (6) that 


$$
\begin{aligned}
2 \sum S_{1} & {\left[d_{2} \equiv \delta_{3}(\bmod 2), \delta_{2} \equiv d_{3}(\bmod 2)\right] } \\
= & \sum\left\{S_{2}\left[d_{2} \equiv \delta_{3}(\bmod 2), \delta_{2} \equiv d_{3}(\bmod 2)\right]\right. \\
& -S_{2}\left[d_{2} \equiv \delta_{2} \equiv d_{3} \equiv \delta_{3}(\bmod 2), d_{2}=d_{3}\right] \\
& \left.+2 S_{2}\left[d_{2} \equiv \delta_{3}(\bmod 2), d_{3} \equiv 0(\bmod 2), d_{3}>d_{2}, \delta_{2}=0\right]\right\} .
\end{aligned}
$$

This is the first fundamental formula.

As a consequence of the correspondences (4) and

(8) $d_{1}^{\prime}=d_{1}+d_{2}, \delta_{1}^{\prime}=\delta_{1}, d_{2}^{\prime}=d_{2}, \delta_{2}^{\prime}=\delta_{2}-\delta_{1}, d_{3}^{\prime}=d_{3}, \delta_{3}^{\prime}=\delta_{3}$,

it is evident that

$$
\begin{aligned}
& \sum S_{4} {\left[d_{1} \not \equiv \delta_{3}(\bmod 2), \delta_{2} \not \equiv d_{3}(\bmod 2)\right] } \\
&=\sum S_{4}^{\prime}\left[d_{1}^{\prime} \not \equiv \delta_{3}^{\prime}(\bmod 2), \delta_{2}^{\prime} \not \equiv d_{3}^{\prime}(\bmod 2)\right] \\
&=\sum S_{3}\left[d_{1}+d_{2} \not \equiv \delta_{3}(\bmod 2), \delta_{2}-\delta_{1} \not \equiv d_{3}(\bmod 2), \delta_{2}>\delta_{1},\right. \\
&\left.d_{1}+d_{2}>0\right],
\end{aligned}
$$

where, in the last sum, $d_{1}$ is not restricted to positive values. As in the case of (6), $S_{3}$ in the final sum of (9) is invariant for $d_{1}>0$, except that $\delta_{2}>\delta_{1}$ becomes $\delta_{1}>\delta_{2}$, when $d_{1}, \delta_{1}, d_{2}, \delta_{2}$ are replaced by $d_{2}, \delta_{2}, d_{1}, \delta_{1}$, respectively; and for $d_{1}<0, S_{3}$ goes into $-S_{3}$ when $d_{1}, \delta_{1}, d_{2}, \delta_{2}, d_{3}, \delta_{3}$ are replaced by $-\delta_{1},-d_{1}, \delta_{2}, d_{2}, \delta_{3}, d_{3}$ respectively. Because of the form of the expression $S_{4}$ it is also obvious that the first sum in (9) is zero. From these results we obtain the second fundamental formula

$$
\begin{aligned}
& \sum S_{3}\left[d_{1}+d_{2} \not \equiv \delta_{3}(\bmod 2), \delta_{2}-\delta_{1} \not \equiv d_{3}(\bmod 2)\right] \\
& =\sum\left\{S_{3}\left[\delta_{3} \not \equiv d_{1}+d_{2}(\bmod 2), d_{3} \neq \equiv 0(\bmod 2), \delta_{1}=\delta_{2}\right]\right. \\
& \left.\quad-2 S_{3}\left[d_{2} \not \equiv \delta_{3}(\bmod 2), \delta_{2}-\delta_{1} \not \equiv d_{3}(\bmod 2), \delta_{2}>\delta_{1}, d_{1}=0\right]\right\} .
\end{aligned}
$$

4. Formula $(Q)$. In each of (7) and (10) let us choose $n=m$ (odd), $F(x, y)=\phi(x, y)$ for $x \equiv y \equiv 0(\bmod 2)$, and $F(x, y)=0$ otherwise. It is then clear that $\phi(x, y)$ is subject to the same conditions as $F(x, y)$ in (3) and, after slight changes in notation, that the left members of (7) and (10) are equal. Elimination of this common sum gives

$$
\begin{aligned}
& \sum \bar{S}_{2}\left[d_{i} \equiv \delta_{i} \equiv 1(\bmod 2)(i=1,2,3)\right] \\
& =\sum\left\{\bar{S}_{2}\left[d_{i} \equiv \delta_{i} \equiv 1(\bmod 2)(i=1,2,3), d_{2}=d_{3}\right]\right. \\
& \quad-2 \bar{S}_{3}\left[d_{2} \neq \delta_{3}(\bmod 2), \delta_{2}-\delta_{1} \neq d_{3}(\bmod 2),\right. \\
& \left.\left.\delta_{2}>\delta_{1}, d_{1}=0\right]\right\} .
\end{aligned}
$$

In (11) and later expressions, it is to be remembered that $x$ and $y$ in $\phi(x, y)$ are both even integers. 
It will now be proved that the sum in the right member of (11) is zero. On placing $\Delta_{2}=\delta_{2}+\delta_{3}$ we have

$$
\begin{aligned}
& \sum \bar{S}_{2}\left[d_{i} \equiv \delta_{i} \equiv 1(\bmod 2)(i=1,2,3), d_{2}=d_{3}\right] \\
& =2 \sum^{\prime} \sum{ }_{J} \Delta_{2} \phi\left(\delta_{1}-J, \delta_{1}+\Delta_{2}-J\right)\left[d_{1} \equiv \delta_{1} \equiv d_{2}\right. \\
& \left.\equiv J \equiv 1 \neq \Delta_{2}(\bmod 2), \Delta_{2}>J\right],
\end{aligned}
$$

where $\sum_{J}$ indicates the summation over all positive odd integers $J$, in this case less than $\Delta_{2}$, and $\sum^{\prime}$ indicates the summation over positive integral solutions $\left(d_{1}, \delta_{1}, d_{2}, \Delta_{2}\right)$ for $m$ in the form $m=d_{1} \delta_{1}+d_{2} \Delta_{2}$, with $d_{1} \equiv \delta_{1} \equiv d_{2} \not \equiv \Delta_{2} \equiv 0(\bmod .2)$. Now

$$
\begin{aligned}
& 2 \sum^{\prime} \sum_{J} \Delta_{2} \phi\left(\delta_{1}-J, \delta_{1}+\Delta_{2}-J\right)\left[\Delta_{2}>J\right] \\
&=2 \sum^{\prime} \sum_{J}\left\{\Delta_{2} \phi\left(\delta_{1}-J, \delta_{1}+\Delta_{2}-J\right)\right.\left.-\Delta_{2} \phi\left(\delta_{1}-J, \delta_{1}+\Delta_{2}-J\right)\left[\Delta_{2}<J\right]\right\} \\
&=2 \sum^{\prime} \sum_{J} \Delta_{2}\left\{\phi\left(\delta_{1}-J, \delta_{1}+\Delta_{2}-J\right)\right. \\
&\left.-\phi\left(\delta_{1}-\Delta_{2}-J, \delta_{1}-J\right)\right\}
\end{aligned}
$$

the last term being obtained from that for which $\Delta_{2}$ is less than $J$ by first replacing $J$ by $\Delta_{2}+k$ and then changing $k$ into $J$.

In case $\delta_{1}>J$ the final sum in (12) is equal to

$2 \sum \bar{S}_{8}\left[d_{2} \not \equiv \delta_{3}(\bmod 2), \delta_{2}-\delta_{1} \neq \equiv d_{3}(\bmod 2), \delta_{2}>\delta_{1}, d_{1}=0\right]$.

In case $\delta_{1}<J$ let us replace $J$ by $\delta_{1}+2 k, k$ a positive integer. Then the final sum in (12) becomes

$$
\begin{gathered}
2 \sum^{\prime} \sum_{k} \Delta_{2}\left\{\phi\left(-2 k, \Delta_{2}-2 k\right)-\phi\left(-\Delta_{2}-2 k,-2 k\right)\right\} \\
=2 \sum^{\prime} \sum_{k} \Delta_{2}\left\{\phi\left(2 k, \Delta_{2}+2 k\right)+\phi\left(\Delta_{2}-2 k, 2 k\right)\right\} .
\end{gathered}
$$

Now, for $\Delta_{2}>2 k$ and hence for $2 k=\Delta_{2}-2 i, i$ a positive integer, $\sum^{\prime} \sum_{k} \Delta_{2} \phi\left(\Delta_{2}-2 k, 2 k\right)\left[\Delta_{2}>2 k\right]$

$$
=-\sum^{\prime} \sum_{i} \Delta_{2} \phi\left(\Delta_{2}-2 i, 2 i\right)\left[\Delta_{2}>2 i\right],
$$

so that

$$
\sum^{\prime} \sum_{k} \Delta_{2} \phi\left(\Delta_{2}-2 k, 2 k\right)\left[\Delta_{2}>2 k\right]=0 .
$$

On the other hand, for $\Delta_{2}<2 k$ and hence for $2 k=\Delta_{2}+2 i$,

$$
\begin{aligned}
\sum^{\prime} \sum_{k} \Delta_{2} \phi\left(\Delta_{2}-2 k, 2 k\right)\left[\Delta_{2}\right. & <2 k] \\
& =\sum^{\prime} \sum_{i} \Delta_{2} \phi\left(-2 i, \Delta_{2}+2 i\right)[i>0] \\
& =-\sum^{\prime} \sum_{k} \Delta_{2} \phi\left(2 k, \Delta_{2}+2 k\right)[k>0] .
\end{aligned}
$$

Since the right-hand sum in (13) is thus zero, it follows from (11) that 


$$
\sum \bar{S}_{2}\left[d_{i} \equiv \delta_{i} \equiv 1(\bmod 2)(i=1,2,3)\right]=0 .
$$

As a consequence of (3), formula (14) reduces to

$$
\begin{aligned}
\sum d_{3}\left\{\phi\left(d_{1}+d_{3}, d_{1}+d_{2}\right)+\phi\left(d_{1}-d_{3}, d_{1}+d_{2}\right)\right\} \\
=\sum d_{3}\left\{\phi\left(d_{1}+d_{3}, d_{1}-d_{2}\right)+\phi\left(d_{1}-d_{3}, d_{1}-d_{2}\right)\right\},
\end{aligned}
$$

where the sums are taken over all solutions of $m$ in the form $d_{1} \delta_{1}+d_{2} \delta_{2}$ $+d_{3} \delta_{3}$ with $d_{i} \equiv \delta_{i} \equiv 1(\bmod 2)$ for $i=1,2,3$, and where $\phi(x, y)$ satisfies the same conditions as $F(x, y)$ in (3). This is Liouville's (Q).

5. A more general formula. Formula $(Q)$ is only one of several arithmetical identities which we have established. Each of (7) and (10), for example, is also an identity of the Liouville type. Further formulas are obtained from (7) and (10) by deleting the congruence relations from the restrictions in the square brackets.

In $\$ 2$ let us re-define the notation by placing

$$
\begin{aligned}
S_{1}= & F\left(d_{3}, d_{1}+d_{2}+d_{3}, d_{1}+d_{2}\right)-F\left(d_{3}, d_{1}-d_{2}-d_{3}, d_{1}-d_{2}\right) \\
& +F\left(\delta_{2}, \delta_{1}-\delta_{3}, \delta_{1}+\delta_{2}-\delta_{3}\right)-F\left(\delta_{2}, \delta_{1}+\delta_{3}, \delta_{1}-\delta_{2}+\delta_{3}\right) \\
S_{2}= & F\left(d_{3}-d_{2}, d_{1}+d_{3}, d_{1}+d_{2}\right)-F\left(d_{3}-d_{2}, d_{1}-d_{3}, d_{1}-d_{2}\right) \\
& +F\left(\delta_{3}+\delta_{2}, \delta_{1}-\delta_{3}, \delta_{1}+\delta_{2}\right)-F\left(\delta_{2}+\delta_{3}, \delta_{1}+\delta_{3}, \delta_{1}-\delta_{2}\right) \\
S_{3}= & F\left(d_{3}, d_{1}+d_{2}+d_{3}, d_{1}+d_{2}\right)-F\left(d_{3}, d_{1}+d_{2}, d_{1}+d_{2}-d_{3}\right) \\
& +F\left(\delta_{3}, \delta_{2}-\delta_{1}, \delta_{2}-\delta_{1}+\delta_{3}\right)-F\left(\delta_{3}, \delta_{2}-\delta_{1}-\delta_{3}, \delta_{2}-\delta_{1}\right) \\
S_{4}= & F\left(d_{3}, d_{1}+d_{3}, d_{1}\right)-F\left(d_{3}, d_{1}, d_{1}-d_{3}\right) \\
& +F\left(\delta_{3}, \delta_{2}, \delta_{2}+\delta_{3}\right)-F\left(\delta_{3}, \delta_{2}-\delta_{3}, \delta_{2}\right)
\end{aligned}
$$

where

$$
\begin{aligned}
F(w,-x, y) & =-F(w, x, y)=F(w, x,-y) \\
& =F(w, y, x)=F(-w, x, y), \\
F(0, x, y) & =0=F(w, 0, y)=F(w, x, 0) .
\end{aligned}
$$

It is not difficult to see that (7) and (10) still hold. If, further, we let $n=m$ (odd) in each of these formulas, choose $F(w, x, y)=\psi(w, x, y)$ for $w \equiv x \equiv y \equiv 0(\bmod 2)$ and $F(w, x, y)=0$ otherwise, and follow the procedure outlined in $\$ 4$, we obtain the more general formula

$$
\begin{aligned}
& \sum\left\{\psi\left(d_{3}-d_{2}, d_{1}+d_{8}, d_{1}+d_{2}\right)+\psi\left(d_{3}+d_{2}, d_{1}-d_{3}, d_{1}+d_{2}\right)\right\} \\
& =\sum\left\{\psi\left(d_{3}+d_{2}, d_{1}+d_{3}, d_{1}-d_{2}\right)+\psi\left(d_{3}-d_{2}, d_{1}-d_{3}, d_{1}-d_{2}\right)\right\}
\end{aligned}
$$

where $\psi(w, x, y)$ is subject to the same conditions as $F(w, x, y)$ in (15). 
Liouville's $(Q)$ is a special case of (15) for the choice $\psi(w, x, y)$ $=w \phi(x, y)$.

\section{REFERENCES}

1. L. E. Dickson, History of the theory of numbers, vol. 2, p. 339.

2. J. Liouville, Sur quelques formules générales qui peuvent être utiles dans la thêrie des nombres, Journal de Mathématiques (2) vol. 3 (1858) pp. 325-336.

3. E. T. Bell, An arithmetic formula of Liouville, Bull. Amer. Math. Soc. vol. 27 (1921) pp. 330-332.

The University of British Columbia 